\title{
Amiodarone-induced vortex keratopathy at a low maintenance dose
}

\author{
Deepti Mahajan, Anil Verma, Rajesh Sharma \\ Department of Ophthalmology, Rajendra Prasad Medical College, Tanda, India
}

\begin{abstract}
Vortex keratopathy is a common side effect of amiodarone, which is a class III antiarrhythmic agent. We describe a 50-year-old man who developed vortex keratopahy with amidarone $200 \mathrm{mg}$ BD for atrial fibrillation since two years. The daily (400 mg/day) and cumulative dose $(100 \mathrm{~g})$ combined with the length of therapy is associated with the toxicity. Toxic effects may also be observed at lower maintenance doses, as observed in this patient. This case indicates that multi-organ toxicity due to amiodarone may develop even with short-term use and a low maintenance dose. Having been off the medication for two months, it is expected that the deposition pattern will diminish, as is the case for the vast majority of patients.
\end{abstract}

Keywords: amiodarone, keratopathy, vortex

Vortex keratopathy is a common side effect of amiodarone, which is a class III antiarrhythmic agent effective against all types of tachyarrhythmias. It is well known to cause toxicity affecting the lungs, thyroid gland, liver, eyes, skin and nerves. The most common ocular findings that have been reported due to amiodarone are corneal, lens opacities and optic neuropathy.

We describe a 50-year-old man who was referred by the medicine department after he reported glare in his eyes for a few weeks. He was taking amiodarone $200 \mathrm{mg} \mathrm{BD}$ for atrial fibrillation since 2 years. His best-corrected visual acuity was $6 / 6$ bilaterally. Slit-lamp examination of the anterior segment showed a whorl-like pattern of corneal epithelial deposits bilaterally, characterizing amiodarone-induced vortex keratopathy (also called cornea verticillata). Other ocular structures were normal (Fig. 1). Fundus examination revealed a normal fundus in the right eye and temporal pallor in the left eye (Fig. 2).

The ocular effect of amiodarone is vortex keratopathy creating a whorl-like pattern by producing lysosomal deposits in the basal epithelial layer., ${ }^{2,3}$ The whorl-like pattern which was firstly described by Fleischer in 1910 is characterized as powdery, white, yellow or brown corneal opacities beneath the cornea apex. Two mechanisms describe the production of the pattern. The first is that

Correspondence: Dr. Deepti Mahajan, Arunodya, Cemetry Road, Sanjauli, Shimla, Himachal Pradesh, India 171006.

E-mail:mahajandeepti22@gmail.com 


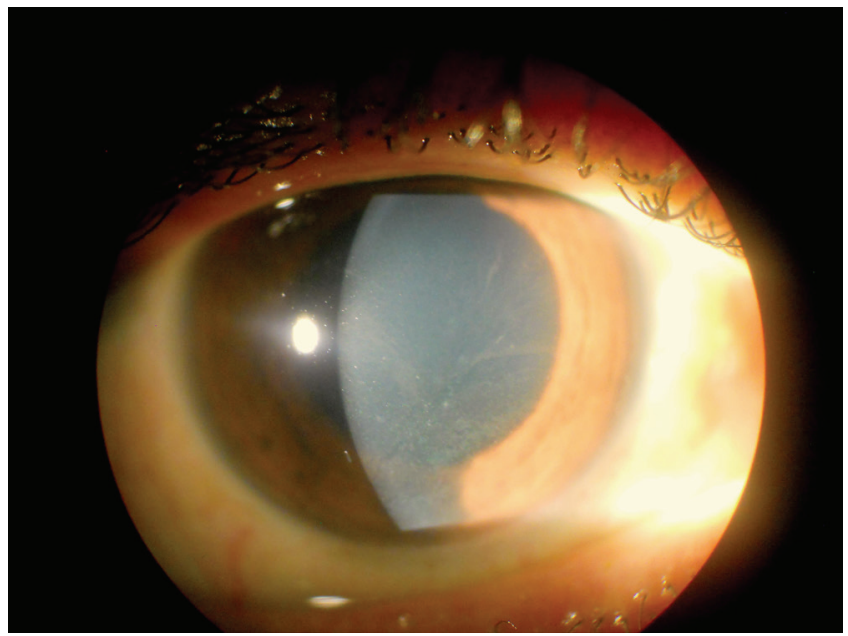

Fig. 1. Amiodarone deposits in the corneal epithelium.

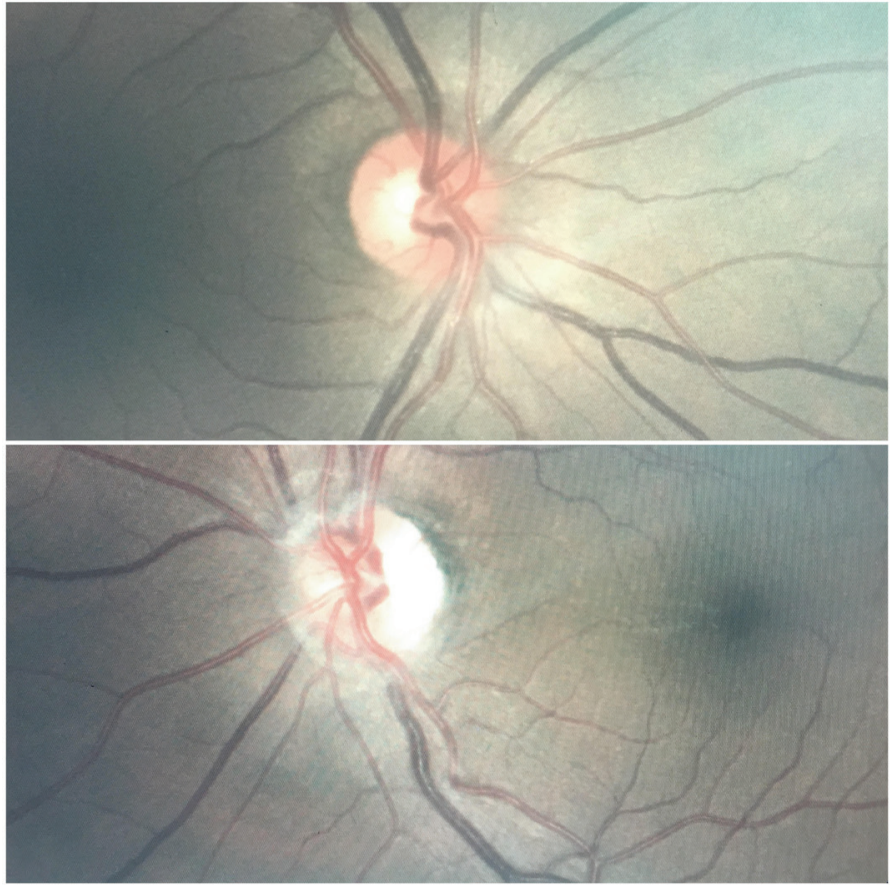

Fig. 2. Fundus photograph of the patient. 
the predominant horizontal line across the lower two-thirds of the cornea corresponds to the line of lid closure and that the deposits result directly from the concentration of the drug in the tears. ${ }^{4}$ Another theory is that the lines are a manifestation of the centripetal trajectory of migrating epithelial cells from a non-uniform distribution of stem cells at the limbus. The direction of movement is thought to follow a pattern similar to that of water flowing down a plug hole, with the point of the vortex in the midline at the junction of the lower and middle third of the cornea. ${ }^{5}$

The daily ( $400 \mathrm{mg} /$ day) and cumulative dose $(100 \mathrm{~g})$ combined with the length of therapy is associated with the toxicity. Toxic effects may also be observed at lower maintenance doses, as observed in this patient. ${ }^{2}$ This case indicates that multi-organ toxicity due to amiodarone may develop even with short-term use and a low maintenance dose. Laboratory studies including liver and thyroid functions should be checked every 6 months and ocular examination should be done regularly to detect ocular side effects to prevent irreversible ocular damages in patients taking amiodarone. Hence amiodarone should be used at lowest possible doses. Having been off the medication for two months, it is expected that the deposition pattern will diminish, as is the case for the vast majority of patients.

\section{Acknowledgement}

I would like to thank the entire Ophthalmology Department which worked as a team for doing various procedures for the patients.

\section{References}

1. Gittinger JW, Jr, Asdourian GK. Papillopathy caused by amiodarone. Arch Ophthalmol. 1987;105:349-351.

2. Hollander DA, Aldave AJ. Drug-induced corneal complications. Curr Opin Ophthalmol. 2004;15:541-548.

3. D'Amico DJ, Kenyon KR, Ruskin JN. Amiodarone keratopathy: drug-induced lipid storage disease. Arch Ophthalmol. 1981;99:257-261.

4. Flach AJ, Dolan BJ, Sudduth B, Weddell J. Amiodarone-induced lens opacities. Arch Ophthalmol. 1983;101:1554-1556.

5. Uçakhan OO, Kanpolat A, Ylmaz N, Ozkan M. Amiodarone keratopathy: an in vivo confocal microscopy study. Eye Contact Lens. 2005;31:148-157. 